\title{
AIAA 2001-2819 \\ Effective Thermal Conductivity of High Porosity Open Cell Nickel Foam
}

\author{
Alan D. Sullins \\ The George Washington University \\ Hampton, VA \\ Kamran Daryabeigi \\ NASA Langley Research Center \\ Hampton, VA
}

Joint Institute for Advancement of Flight Sciences

\section{$35^{\text {th }}$ AIAA Thermophysics Conference}

11-14 June 2001 / Anaheim, CA 
AIAA 2001-2819

\section{EFFECTIVE THERMAL CONDUCTIVITY OF HIGH POROSITY OPEN CELL NICKEL FOAM}

\author{
Alan D. Sullins* \\ The George Washington University \\ Joint Institute for Advancement of Flight Sciences \\ Hampton, Virginia
}

\author{
Kamran Daryabeigi ${ }^{\dagger}$ \\ NASA Langley Research Center \\ Hampton, Virginia
}

\begin{abstract}
$\underline{\text { Abstract }}$
The effective thermal conductivity of high-porosity open cell nickel foam samples was measured over a wide range of temperatures and pressures using a standard steady-state technique. The samples, measuring $23.8 \mathrm{~mm}, 18.7 \mathrm{~mm}$, and $13.6 \mathrm{~mm}$ in thickness, were constructed with layers of $1.7 \mathrm{~mm}$ thick foam with a porosity of 0.968 . Tests were conducted with the specimens subjected to temperature differences of 100 to $1000 \mathrm{~K}$ across the thickness and at environmental pressures of $10^{-4}$ to $750 \mathrm{~mm} \mathrm{Hg}$. All test were conducted in a gaseous nitrogen environment. A one-dimensional finite volume numerical model was developed to model combined radiation/conduction heat transfer in the foam. The radiation heat transfer was modeled using the two-flux approximation. Solid and gas conduction were modeled using standard techniques for high porosity media. A parameter estimation technique was used in conjunction with the measured and predicted thermal conductivities at pressures of $10^{-4}$ and $750 \mathrm{~mm} \mathrm{Hg}$ to determine the extinction coefficient, albedo of scattering, and weighting factors for modeling the conduction thermal conductivity. The measured and predicted conductivities over the intermediate pressure values differed by 13 percent.
\end{abstract}

\section{Introduction}

Thermal Protection Systems (TPS) are currently under development for a wide range of aerospace applications. Metallic foam has been considered as the insulating material of the TPS on reusable launch vehicles. Another application is to use the metallic foam as part of an integrated structure that serves as the launch vehicle's primary structure and thermal protection system. However, limited information is available on the thermal properties of metallic foams under the environmental conditions to which re-entry type vehicles are exposed. Earth re-entry typically produces aerodynamic heating to a surface to temperatures as high as $1000^{\circ} \mathrm{C}$ in a pressure range from $10^{-2}$ to $760 \mathrm{~mm} \mathrm{Hg}$.

Metallic foams have been recently investigated for various applications. Aluminum foams have been utilized in the automobile industry for their crashworthy properties. ${ }^{2}$ Nickel foams have been used to improve the performance in high-power batteries. Foam-based nickel metal hydride batteries currently compete with the more expensive lithium ion batteries for lightweight cordless electronics. ${ }^{3}$

There has been extensive work in both experimental and analytical modeling of heat transfer in porous media. Kaviany has provided a comprehensive review of heat transfer in general porous material. ${ }^{4}$ Lee and Cunnington have provided an extensive review of conduction and radiation heat transfer in high porosity fibrous insulation. ${ }^{5}$ Glicksman has reviewed heat transfer in polymeric foams. ${ }^{6}$ Gibson and Ashby discussed thermal properties of foams in their comprehensive work on cellular solids. ${ }^{7}$ Ballis, et. al., modeled heat transfer in open cell carbon foams and determined radiation as the primary source of heat transfer for temperatures above $1000 \mathrm{~K}^{8}$ They used the optically thick approximation for modeling radiation with a weighted spectral extinction coefficient to account for anisotropic scattering. In addition, they used linear superposition of solid conduction, gas conduction, and radiation thermal conductivities. Their work was limited to atmospheric pressure. There has been limited work on metallic foams. Calmidi and

\footnotetext{
* Graduate Research Scholar Assistant, The George Washington University/JIAFS.

Aerospace Engineer, Senior Member, AIAA

Copyright (C) 2001 by the American Institute of Aeronautics and Astronautics, Inc. All rights reserved.
} 
Mahajan measured the thermal conductivity of aluminum foams at temperatures up to $75^{\circ} \mathrm{C}$ with a $15^{\circ} \mathrm{C}$ temperature difference maintained across the specimen. ${ }^{9}$ Under these experimental conditions nonlinear effects such as natural convection and radiation were determined to be negligible. Their work was also limited to atmospheric pressure. They modeled the heat transfer as combined gas and solid conduction and formulated an empirical correlation for the thermal conductivity in the aluminum foam. Bhattachrya, et. al., performed further refinements on the gas/solid conduction modeling of Calmidi and Mahajan, again ignoring radiation heat transfer. ${ }^{10}$ They showed that the effective thermal conductivity had a strong dependence on the porosity and the particular geometrical configurations of the intersection of the struts in the foam.

The objective of the present study was to investigate heat transfer in metallic foams over a wide range of pressures and temperatures where solid conduction, gas conduction, and radiation were the three modes of heat transfer. Therefore, the effective thermal conductivity of the nickel foam was measured in the temperature range of 300 to $1300 \mathrm{~K}$ and environmental pressures of $10^{-4}$ to $750 \mathrm{~mm} \mathrm{Hg}$. The two-flux approximation was used to model the radiation heat transfer through the nickel foam. A combined conduction formulation based on the superposition of solid conduction and gas conduction was used to model conduction heat transfer. The temperature dependent gas conduction model was applicable over the rarefied, transition, and continuum gas transport regimes. Inverse heat transfer methods were used to determine the parameters needed in the heat transfer model.

\section{Metallic Nickel Foam}

The metallic nickel foam used in this study is commercially available. It is manufactured in bulk for use in a variety of applications. In the manufacturing process, polyurethane foam is used as a template. A proprietary chemical vapor decomposition process coats the surface of the template with nickel. The material is annealed at around $1800^{\circ} \mathrm{C}$, causing evaporation of the polyurethane core. Photomicrograph images of the foam produced by an electron scanning microscope with magnification factors of 75 and 750 are shown in Figures 1 and 2, respectively. As seen from the images, the foam has an open cell structure with randomly oriented struts. In addition, the struts are themselves hollow, left void due to the removal of the polyurethane template. The metallic foam used in this study is $99.98 \%$ nickel by

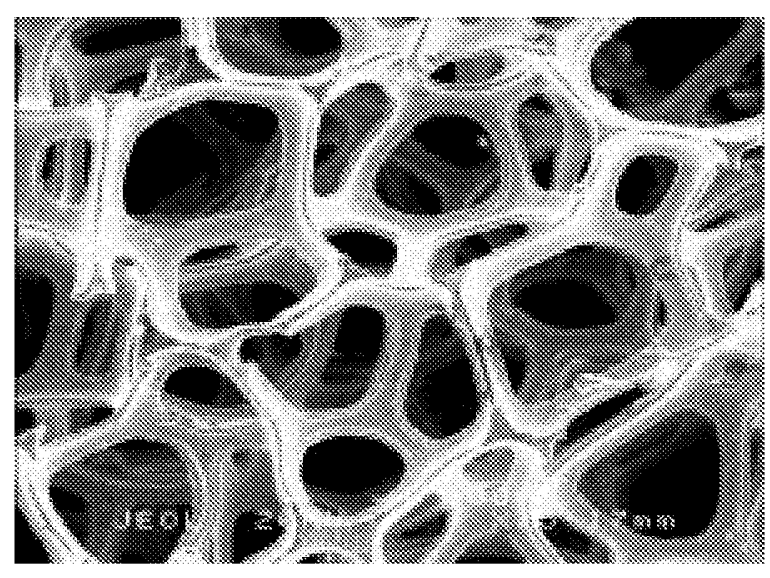

Figure 1. Photomicrograph of nickel foam (magnification factor $=75$ ).

composition with porosity of 0.968 . The foam is 1.7 $\mathrm{mm}$ thick and has a density of $290 \mathrm{~kg} / \mathrm{m}^{3}$ at room temperature and atmospheric pressure. Although it would have been ideal to have samples of different thicknesses, the $1.7 \mathrm{~mm}$ thick foam was the only material available that provided the desired porosity and composition. As a result, three samples were constructed utilizing 14, 11, and 8 layers of the metallic foam. The corresponding thicknesses of the samples were $23.8 \mathrm{~mm}, 18.7 \mathrm{~mm}$, and $13.6 \mathrm{~mm}$, respectively.

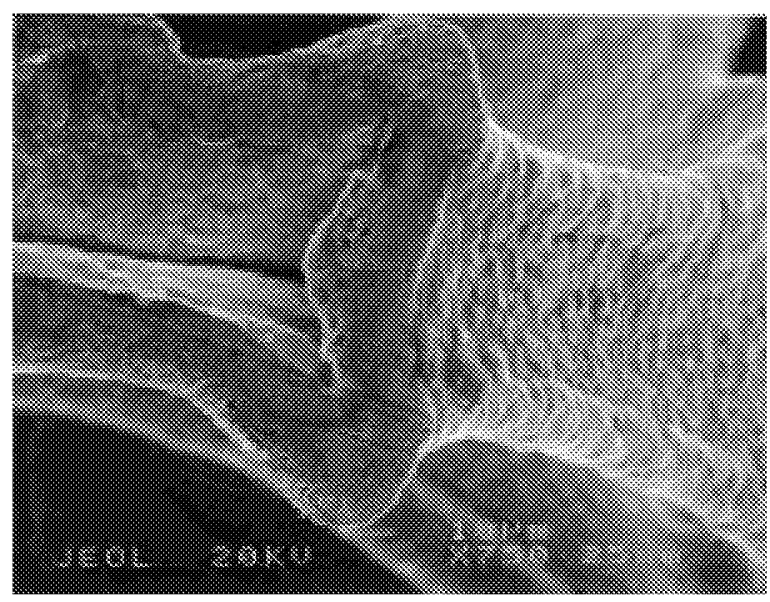

Figure 2. Photomicrograph of nickel foam ( magnification factor $=\mathbf{7 5 0})$.

\section{Experimental Procedure}

The apparatus used to determine the effective thermal conductivity of the foam has been used in previous work to investigate heat transfer through fibrous insulation. ${ }^{11}$ The sample was placed between a radiantly heated septum plate that can reach 
temperatures as high as $1300 \mathrm{~K}$ and a water-cooled plate maintained at room temperature. The watercooled plate was placed on the bottom so the direction of the applied heat flux was opposite to the local gravity vector thus eliminating natural convection.

Thermocouples were placed within the septum plate and within the water-cooled plate to measure the plate temperatures. Heat flux gauges, located on the watercooled plate, measured the flux of heat energy flowing through the sample. The entire apparatus was housed in a $1.5 \times 1.5$ meter vacuum chamber. Upon insertion of the foam sample into the test apparatus, shown in Figure 3, the gas inside the vacuum chamber was removed. Once high vacuum was achieved, nitrogen gas was used to regulate the gas pressure within the chamber. The primary reason nitrogen gas was selected for use in this experiment was to eliminate the oxidation of the foam at higher exposure temperatures.

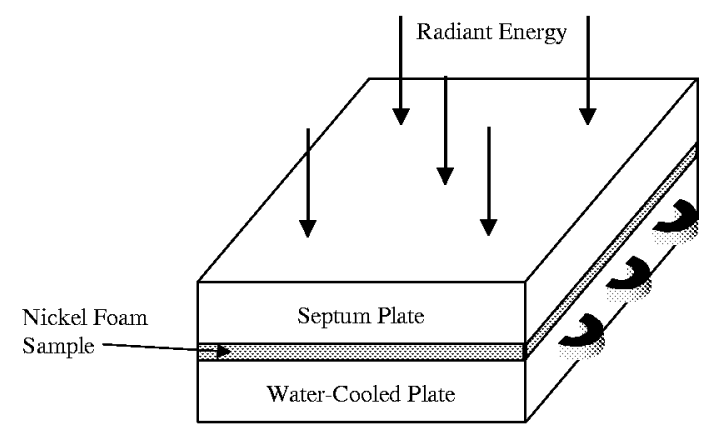

Figure 3. Schematic of experimental apparatus.

Each measurement consisted of setting the septum plate to the desired temperature, varying the nitrogen gas to the desired pressure, and allowing both to reach a steady state condition. Additionally, the time rate of change of the heat flux through the sample was monitored until it approached zero. Once steady-state environmental conditions were achieved, the temperatures of the septum plate and water-cooled plate and the heat fluxes were recorded. The effective thermal conductivity of the metallic foam, $k_{\text {eff }}$, was determined using Fourier's law

$$
k_{\text {eff }}=\frac{q \cdot L}{T_{\text {Hor }}-T_{\text {Cold }}}
$$

where $\mathrm{L}$ is the thickness of the sample, $T_{H o t}$ is the temperature on the hot side of the sample, $T_{\text {Cold }}$ is the temperature on the cold side of the sample, and $q$ is the steady-state heat flux through the sample. Lateral heat losses due to edge effects were neglected by restricting measurements to the inner $101.6 \mathrm{~mm}$ square region of the $304.8 \mathrm{~mm}$ square sample. Within the inner region, measurements were taken at four different locations. Values were recorded at a 5-second interval for two minutes. The average of the measurements of the four spatial locations over the two-minute interval was taken to be the steady-state measurement. Steady-state measurements were taken at 7 different nominal septum plate temperatures (from $100^{\circ} \mathrm{C}$ to $1000^{\circ} \mathrm{C}$ ) and 10 different gas pressures (from $10^{-4}$ to $750 \mathrm{~mm} \mathrm{Hg}$ ).

\section{Analytical Model}

Existing techniques were utilized, modified, and combined to model the effective thermal conductivity of the metallic foam in the temperature and pressure range covered by the experimental data., ${ }^{1,4-11,13}$

The conservation of energy principle for a control volume, states that the time rate of increase in the energy stored in the volume plus the net rate at which energy is conducted out through the surface of the volume is equal to zero. The statement can be written in integral form as

$$
\iiint_{R} \rho \cdot c \cdot \frac{\partial T}{\partial t} d R+\oiint_{S} \mathbf{q} \cdot \mathbf{n} d S=0
$$

where $\rho$ is the density, $c$ is the specific heat of the material, $T$ is the absolute temperature, $q$ is the heat flux vector, and $\mathbf{n}$ is the normal to the control volume surface. $^{12}$

When the heat flux vector is unidirectional and parallel to the normal of the surface of the control volume (heat flow in one direction only), Equation 2 reduces to

$$
\rho \cdot c \cdot \Delta x \cdot \frac{\partial T}{\partial t}+q_{\text {out }}=q_{\text {in }}
$$

The heat flux into the volume, $q_{\text {in }}$, and the heat flux out of the volume, $q_{\text {out }}$, take the general form

$$
q=q_{\text {cond }}+q_{\text {rad }}
$$

where $q_{\text {cond }}$ is the amount of heat transferred by thermal conduction and $q_{\text {rad }}$ is the amount of heat transferred by radiation.

Analytical models for the heat transferred by conduction and radiation are discussed and quantified. The one-dimensional finite volume numerical method is presented. The nonlinear parameter estimation method used to determine intrinsic material properties needed in the analytical formulation is also briefly described. 


\section{$\underline{\text { Conduction }}$}

The conduction of heat energy through the foam is a combination of conduction through the solid struts of the foam and conduction through the gas within the void of the foam. The heat flow due to conduction is given by

$$
q_{\text {cond }}=-k \cdot \frac{\partial T}{\partial x}
$$

where $k$ is the combined conduction thermal conductivity and $x$ is the independent spatial variable in the direction of the sample thickness. Several theories have been developed to describe the combined conduction thermal conductivity in terms of the conductivity of the solid material that makes up the foam and the conductivity of the gas that fills the voids. The limiting case, a parallel arrangement, is based on the fraction of cross sectional area responsible for the conduction of heat energy. ${ }^{13}$ The porosity, $\varepsilon$, defined as the total void volume divided by the total volume of the foam, is used as the fraction of the area that will conduct heat due to gas conduction. Then, $(1-\varepsilon)$ is the fraction of the area that will conduct heat due to solid conduction. The parallel arrangement for the combined conduction thermal conductivity is given by

$$
k_{\text {paraltel }}=\varepsilon \cdot k_{\text {gas }}+(1-\varepsilon) \cdot k_{\text {sold }}
$$

where $k_{g a s}$ is the gas thermal conductivity and $k_{\text {solid }}$ is the solid thermal conductivity. Gibson and Ashby assumed that the solid thermal conductivity is related to the thermal conductivity of the strut's parent material through

$$
k_{\text {solid }}=F \cdot k_{\text {bulk }}
$$

where $F$ is the solid conduction efficiency factor which allows for the tortuous path for conduction through the cell walls. ${ }^{7}$ The temperature dependent thermal conductivity of bulk nickel, $k_{\text {bulk }}$, is used in Equation 7. ${ }^{14}$ To correct for overestimation of Equations 6 and 7, Calmidi and Mahajan used the following relationship for modeling heat transfer through aluminum foam

$$
k_{\text {cond }}=\varepsilon \cdot k_{\text {gas }}+(1-\varepsilon)^{m} \cdot D \cdot k_{\text {bulk }}
$$

and they found that $D=0.181$ and $m=0.763$ produced the best match for their experimental results. ${ }^{9}$ When $D=1$ and $m=3$, Equation 8 takes the same functional form used by Daryabeigi for the combined conduction thermal conductivity in fibrous insulation. ${ }^{11}$

Other models investigated assume the conduction takes place in a combined parallel-series arrangement of the solid and gaseous constituents. The parallel case, given in Equation 6, and the series case given by

$$
k_{\text {series }}=\frac{k_{\text {solid }} \cdot k_{\text {gas }}}{\varepsilon \cdot k_{\text {solid }}+(1-\varepsilon) \cdot k_{\text {gas }}}
$$

are typically combined in one of two ways. One approach assumes the combined conduction thermal conductivity can be obtained by a superposition of the parallel and series arrangements

$$
k=A \cdot k_{\text {parallel }}+(1-A) \cdot k_{\text {series }}
$$

where $A$ is the fraction of heat transfer in parallel mode and $(1-A)$ is the fraction of heat transfer in series mode. ${ }^{15,11}$ The other approach, used by Bhattachrya, et. al. ${ }^{10}$ assumes the combined conduction thermal conductivity is the square root of the sum of the squares of the parallel and series arrangements

$$
k=\sqrt{A \cdot\left(k_{\text {parallet }}\right)^{2}+(1-A) \cdot\left(k_{\text {series }}\right)^{2}}
$$

The superposition of the parallel and series arrangements, Equation 10, with the solid thermal conductivity defined in Equation 7 will be used to model the combined conduction thermal conductivity in this study. Written in terms of the thermal conductivity of the gas and bulk material, the combined conduction thermal conductivity is given by

$$
\begin{aligned}
& k=A \cdot\left[\varepsilon \cdot k_{g a s}+(1-\varepsilon) \cdot F \cdot k_{b u t k}\right] \\
& +(1-A) \cdot\left[\frac{F \cdot k_{b u l k} \cdot k_{g a s}}{\varepsilon \cdot F \cdot k_{b u l k}+(1-\varepsilon) \cdot k_{g a s}}\right]
\end{aligned}
$$

Depending on the values of $A$ and $F$ the forms of the combined conduction thermal conductivity given by Equations 6, 8, 9, and 10 are easily realizable. Setting $A=1$ results in the parallel arrangement, while using $A=0$ produces the series arrangement. The combined conduction thermal conductivity given by Equation 8 is obtained by letting $A=1$ and $F=(1-\varepsilon)^{\mathrm{m}-1} \cdot D$.

The thermal conductivity of the nitrogen gas

$$
k_{g a s}=\frac{k_{g a s}^{*}}{Z}
$$

was based on the gas conduction model used by Daryabeigi, where $k_{g a s}^{*}$ is the temperature dependent thermal conductivity of the gas at atmospheric pressure. ${ }^{1}$ The term in the denominator, $Z$, is defined by

$$
Z=\Phi+\Psi \cdot 2 \cdot \frac{2-\alpha}{\alpha} \cdot \frac{2 \cdot \gamma}{\gamma+1} \cdot \frac{1}{P r} \cdot K n
$$

where $\alpha$ is the thermal accommodation coefficient, $\gamma$ is the specific heat ratio, $\mathrm{Pr}$ is the Prandtl number, and $\mathrm{Kn}$ is the Knudsen number. The parameters $\Phi$ and $\Psi$ depend on the Knudsen number. $\Phi=1, \Psi=0$ for Knudsen number less than 0.01 (continuum regime), $\Phi$ $=1, \Psi=1$ for Knudsen number between 0.01 and 10 (transition regime), and $\Phi=0, \Psi=1$ for Knudsen number greater than 10 (free-molecular regime). The Knudsen number, $K n$, is calculated from 


$$
K n=\frac{\lambda}{\delta}
$$

where $\lambda$ is the gas molecular mean free path and $\delta$ is the characteristic length. The mean free path is given by

$$
\lambda=\frac{K_{B} \cdot T}{\sqrt{2} \cdot \pi \cdot P \cdot d_{g}^{2}}
$$

where $K_{B}$ is the Boltzmann constant, $T$ is the absolute temperature of the medium, $P$ is the pressure, and $d_{g}$ is the gas collision diameter. For gas conduction in porous media, the characteristic length is usually defined as the average linear pore size. ${ }^{4}$ The pore size for metallic foam was assumed to have the same form as the pore size in fibrous insulation, giving a characteristic length of

$$
\delta=\frac{\pi}{4} \cdot \frac{D_{f}}{1-\varepsilon}
$$

where $D_{f}$ is the diameter of the strut. ${ }^{1}$ The specific heat ratio, $\gamma$, for nitrogen gas is $7 / 5$. The thermal accommodation coefficient, $\alpha$, and the Prandtl number, $P r$, for nitrogen gas were taken from Daryabeigi. ${ }^{1}$

\section{Radiation}

Most of the work done on carbon foams and fibrous insulation has used the optically thick approximation for the radiation heat transfer. The optical thickness of the samples studied here was not known a priori; therefore, the use of the optically thick approximation could not be justified. The two-flux approximation, which is applicable over various ranges of optical thickness, was used to determine the amount of heat radiated through the void areas of the foam. ${ }^{1}$ The heat transferred by radiation, $q_{r a d}$, is given by

$$
q_{r a d}=-\frac{1}{3 \cdot \beta} \cdot \frac{\partial G}{\partial x}
$$

where $\beta$ is the extinction coefficient, the fraction of radiation lost to scattering and absorption per unit distance within the participating medium. The incident radiation per unit area, $\mathrm{G}$, is determined from the incident radiation equation,

$$
G-\frac{1}{3 \cdot \beta^{2} \cdot(1-\omega)} \cdot \frac{\partial^{2} G}{\partial x^{2}}=4 \cdot \sigma \cdot T^{4}
$$

where $\omega$ is the albedo of scattering and $\sigma$ is the StefanBoltzmann constant. The incident radiation equation is subjected to the boundary condition at the septum plate of

$$
G-\frac{2}{3 \cdot \beta^{2} \cdot\left(\frac{\varepsilon_{1}}{2-\varepsilon_{1}}\right)} \cdot \frac{\partial G}{\partial x}=4 \cdot \sigma \cdot T_{H o r}^{4}
$$

and the boundary condition at the water-cooled plate of

$$
G+\frac{2}{3 \cdot \beta^{2} \cdot\left(\frac{\varepsilon_{2}}{2-\varepsilon_{2}}\right)} \cdot \frac{\partial G}{\partial x}=4 \cdot \sigma \cdot T_{\text {Cold }}^{4}
$$

where $T_{H o t}$ and $T_{C o l d}$ are the temperatures at the boundary surfaces. The emittance of the septum plate, $\varepsilon_{l}$, is 0.85 and the emittance of the water-cooled plate, $\varepsilon_{2}$, is 0.92 as determined by Daryabeigi. ${ }^{11}$ The assumptions used in this formulation consist of: isotropic scattering, homogeneous and gray medium, diffuse emitting and reflecting surfaces. The assumption of isotropic scattering is not strictly valid, but provides simplification of the governing equations to yield an approximate solution. The extinction coefficient is determined from the specific extinction coefficient, $e$, by

$$
\beta=e \cdot \rho
$$

where $\rho$ is the density of the foam. The specific extinction coefficient and the albedo of scattering are intrinsic properties of the material that are independent of density and must be determined experimentally.

\section{Numerical Finite Volume Formulation}

The analytical solution to the general governing equation of heat flow within the metallic foam is difficult to produce. Therefore, a numerical finite volume scheme was used on the transient heat flow problem. The thickness of the sample was separated into discrete volume elements and the conservation of energy principle was applied to each volume element. The change of the heat stored in each volume element plus the heat flowing out of each volume element was equal to the heat flowing into each volume element. The governing finite volume formulation, based on Equation 3, is given by

$$
\begin{gathered}
\rho \cdot c \cdot \Delta x \cdot \frac{T_{i}^{n+1}-T_{i}^{n}}{\Delta t} \\
+k_{i} \cdot \frac{T_{i}^{n}-T_{i+1}^{n}}{\Delta x}+\frac{1}{3 \cdot \beta_{i}^{n}} \cdot \frac{G_{i}^{n}-G_{i+1}^{n}}{\Delta x} \\
=k_{i-1}^{n} \cdot \frac{T_{i-1}^{n}-T_{i}^{n}}{\Delta x}+\frac{1}{3 \cdot \beta_{i-1}^{n}} \cdot \frac{G_{i-1}^{n}-G_{i}^{n}}{\Delta x}
\end{gathered}
$$

where the superscript denotes the time step and the subscript denotes the spatial step. Boundary conditions applied were constant temperatures corresponding to the measured steady-state temperatures at the top and the bottom of the sample. A linearly varying temperature distribution through the thickness of the samples was selected as the initial condition. At each time step, the incident radiation equation, Equation 19, subjected to the boundary conditions of Equations 20 and 21 , was solved numerically using a finite difference technique to determine values of $\mathrm{G}$ based on the current 
values of the temperatures through the thickness of the sample. The steady-state temperature distribution was found by iterating Equation 23 until the temperature at each volume element did not change with successive iterations. The effective thermal conductivity was calculated from Equation 1 using the temperature difference across the sample and the steady-state total heat flux obtained from the numerical solution.

\section{Parameter Estimation}

The unknown parameters, $A$ and $F$, needed in the combined conduction thermal conductivity, Equation 12 , and the unknown parameters, $e$ and $\omega$, needed in the two-flux approximation for the incident radiation equation were determined using the LevenbergMarquardt method for nonlinear parameter estimation. ${ }^{16}$ The Levenberg-Marquardt method is an iterative procedure based on the minimization of the ordinary least squares norm given by

$$
S(\mathbf{P})=[\mathbf{K}-\mathbf{Y}(\mathbf{P})]^{T}[\mathbf{K}-\mathbf{Y}(\mathbf{P})]
$$

where $\mathbf{P}$ is a vector of $N$ unknown parameters, $\mathbf{K}$ is the vector of $M$ measured values of the effective thermal conductivity, and $\mathbf{Y}(\mathbf{P})$ is the vector of $M$ predicted values of the effective thermal conductivity. The $T$ superscript denotes the transpose. The iterative equations used in the Levenberg-Marquardt method of parameter estimation are

$$
\mathbf{A}\left(\mathbf{P}_{k+1}-\mathbf{P}_{k}\right)=\mathbf{b}
$$

where,

$$
\mathbf{A}=\left(\mathbf{J}_{k}\right)^{T} \mathbf{J}_{k}+\mu_{k} \boldsymbol{\Omega}_{k}
$$

is a $N \times N$ matrix and

$$
\mathbf{b}=\left(\mathbf{J}_{k}\right)^{T}\left[\mathbf{K}-\mathbf{Y}\left(\mathbf{P}_{k}\right)\right]
$$

is a $N$ element vector. The subscript, $k$, denotes the iteration number. The sensitivity or Jacobian matrix coefficients are obtained from

$$
J_{i j}=\frac{\partial Y_{i}}{\partial P_{j}}
$$

where $i=1$ to $M$ and $j=1$ to $N$. The damping parameter, $\mu$, along with the diagonal matrix, $\Omega$, damp oscillations and instabilities that arise out of the illconditioned nature of the problem.

The solution to the system of linear algebraic equations, Equation 25, was used to produce a new set of parameters, $\mathbf{P}_{k+1}$. A negligible change in a successive set of calculated parameters served as the convergence criteria.

\section{Discussion of Results}

The experimental data for the 14, 11, and 8 layer samples are presented and discussed. Then, the assumptions used in the development of the numerical model are explained. Using a subset of experimental data obtained from the 8 layer sample, parameters describing specific intrinsic properties of the foam are obtained. Results generated by the numerical model using the aforementioned intrinsic parameters are presented and compared to the experimental results.

\section{Experimental Results}

Experimentally measured values of the effective thermal conductivity as a function of temperature difference across the sample for the 14,11 , and 8 layer samples are presented in Figures 4, 5, and 6, respectively. Data shown on the graphs are at nitrogen gas pressures of $10^{-4}, 1.0$, and $750 \mathrm{~mm} \mathrm{Hg}$. All three samples behaved in a similar fashion. At low pressure, where gas conduction was negligible, the primary modes of heat transfer were solid conduction and radiation. The magnitude of the effective thermal conductivity was at a minimum at low temperature differences. An increase in the temperature difference across the sample increased the contribution of radiation and the effective thermal conductivity increased as seen on the $10^{-4} \mathrm{~mm} \mathrm{Hg}$ constant pressure curve. An increase in pressure increased the contribution of the gas conduction thus increasing the value of the effective thermal conductivity as evident in the 1.0 and $750 \mathrm{~mm} \mathrm{Hg}$ constant pressure curves.

Detailed error analysis was performed to determine the uncertainty due to the bias and random errors and spatial variations of the measured temperatures and heat fluxes. ${ }^{17}$ The uncertainty in the measured values of the

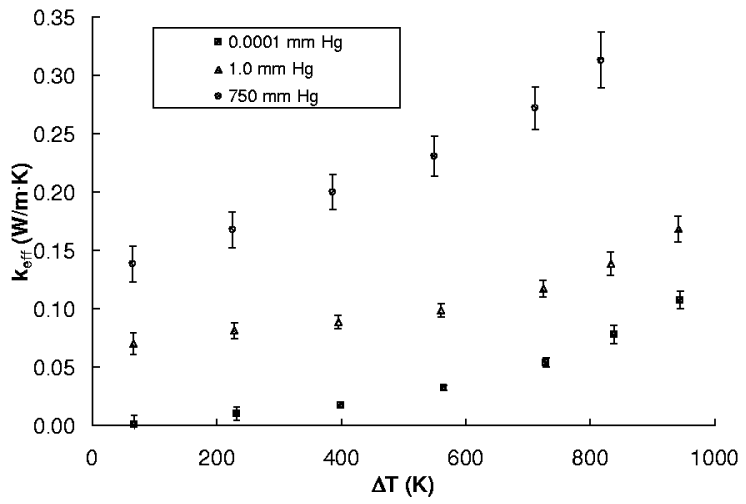

Figure 4. The effective thermal conductivity for the 14 layer sample. 


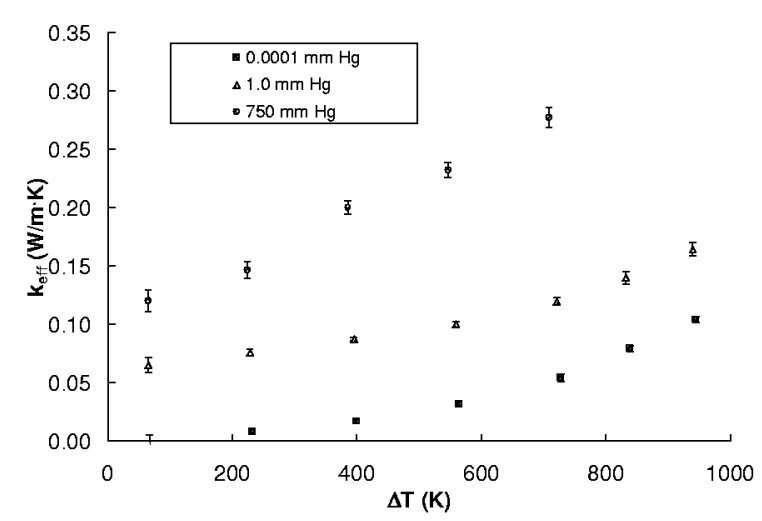

Figure 5. The effective thermal conductivity for the 11 layer sample.

effective thermal conductivity, depicted in Figures 4, 5, and 6 by the error bars, was within a $95 \%$ confidence level. At the lowest temperature differences, the error limits were much larger than the effective thermal conductivity. Neglecting those lowest temperature differences, the error limits for the 14 layer sample varied between 6 percent and 10 percent with an average error limit of 8 percent. Similarly, the 11 layer sample had an average error limit of 4 percent and varied between 2 percent and 9 percent. The 8 layer sample error limits varied between 2 percent and 17 percent with the average being 5 percent.

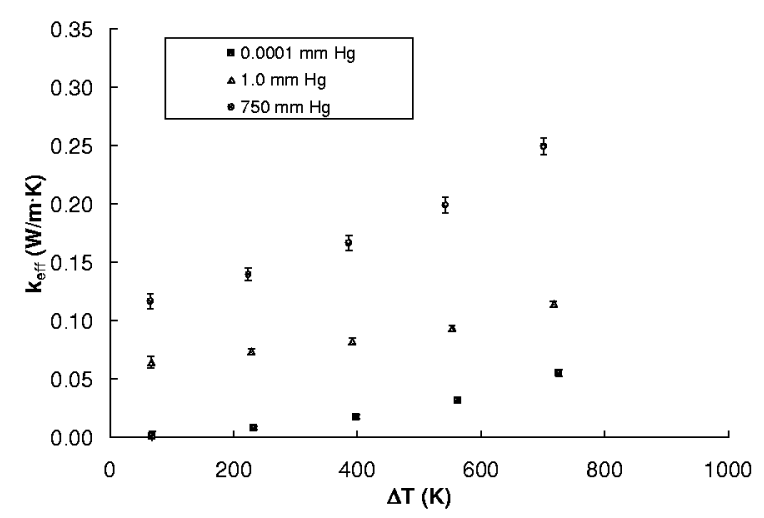

Figure 6. The effective thermal conductivity for the 8 layer sample.

A comparison of the effective thermal conductivity of the different samples is given in Figure 7. At the gas pressure of $10^{-4}$ and $1.0 \mathrm{~mm} \mathrm{Hg}$, the effective thermal conductivity of the three samples was nearly identical and well within the experimental uncertainty range, as expected. The effective thermal conductivity had the same value independent of the thickness of the sample. However, at the gas pressure of $750 \mathrm{~mm} \mathrm{Hg}$, the effective thermal conductivity of the three samples was not nearly as close as desired. The 14 layer and the 8 layer data were significantly different from each other. The first 2 points of the 11 layer matched the 8 layer sample but the remaining points were more consistent with the 14 layer sample. Prolonged exposure of the layers near the septum plate to extreme temperatures throughout the experimental investigation is expected to be the primary source of the deviations. The 8 layer sample was tested first throughout the entire temperature range then used as a core for the 11 layer sample. The 11 layer sample was in turn used as a core for the 14 layer sample.

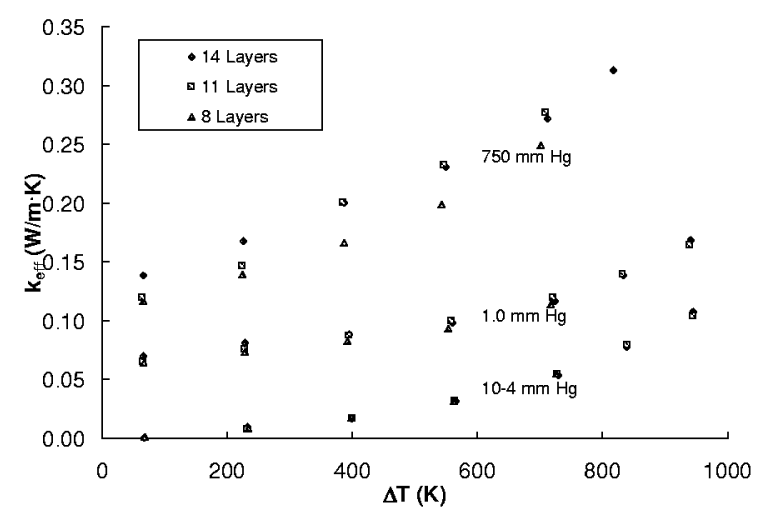

Figure 7. Comparison of the effective thermal conductivity of the three samples.

Inspection of the final sample upon completion of the experimental investigation revealed the 6 layers closest to the septum plate were mechanically interlocked or stuck together. The interlocked layers would be expected to have a higher thermal conductivity than unlocked layers thus contributing to the higher measured values of the effective thermal conductivity for the 11 and 14 layers samples.

Although the thickness of the layered samples was determined to within $0.03 \mathrm{~mm}$, the distance between the septum plate and the water-cooled plate could only be determined to within $0.5 \mathrm{~mm}$ due to the surface variation of the plates. This uncertainty propagated through the detailed error analysis and had a significant effect on the uncertainty of a single measured value. However, the dominant factor in the determination of the error limits depicted in the graphs was the uncertainty due to the spatial variation of the four measurement locations. The uncertainty due to the spatial variation was typically 2 to 3 orders of magnitude greater than the uncertainty of a single measured value. 


\section{Development of the Numerical Model}

For a selected set of boundary conditions (the temperatures at the top and the bottom of the sample), the steady-state temperature distribution and heat flux through the thickness of the samples were obtained from the numerical finite volume formulation of the analytical model of the heat transfer through the foam, Equation 23. Using the temperature difference across the sample defined by the boundary conditions and steady-state heat flux from the numerical solution, the effective thermal conductivity was calculated using Equation 1.

The samples were constructed with an integer number of foam layers. Assuming a uniform distribution of material throughout the sample would simplify the analytical model; however, closer observation of the experimental data at a pressure of $10^{-4}$ and $750 \mathrm{~mm} \mathrm{Hg}$ revealed some interesting trends that could only be explained by incorporating the discrete nature of the layers into the analytical model.

At $10^{-4} \mathrm{~mm} \mathrm{Hg}$, radiation and solid conduction were the main modes of heat transfer. Increasing the pressure to $750 \mathrm{~mm} \mathrm{Hg}$ essentially superimposed gas conduction to the data at $10^{-4} \mathrm{~mm} \mathrm{Hg}$. The difference between the data at the two pressures should be almost equal to the gas thermal conductivity over the temperature range; however, the difference was typically 5 times that of the gas thermal conductivity. Furthermore, the effective thermal conductivities at $10^{4} \mathrm{~mm} \mathrm{Hg}$ were too low to incorporate the contribution of the solid conduction. In other words, a solid conduction efficiency factor, used in Equation 12, could be found to account for the solid conduction at $10^{4} \mathrm{~mm} \mathrm{Hg}$ but the same efficiency factor produced a gross underestimation at $750 \mathrm{~mm} \mathrm{Hg}$. If perfect thermal contact between the different layers in each sample and between the outermost layers and bounding septum and water-cooled plates is assumed, the solid conduction contribution should produce effective thermal conductivity values much higher than what was measured at low pressure.

The only physical explanation for the observed phenomena is that there was a discontinuity in solid conduction between the sample layers and between the outermost layers and the bounding plates (septum and water-cooled). The only conduction mechanism between the sample layers and between the outermost layers and the bounding plates was gas conduction. Thus, at $10^{-4} \mathrm{~mm} \mathrm{Hg}$, since gas conduction was negligible, there was little appreciable transfer of heat between the sample layers by conduction, and the net heat flux through the sample was due mostly to radiation. As gas pressure increased, heat was conducted between the sample layers and between the outermost layers and the bounding plates by gas conduction.

This discontinuity in solid conduction was modeled by placing a small gap between the sample layers and between the outermost layers and the bounding plates. A gap spacing of $0.01 \mathrm{~mm}$ was used. The gap contained only gas, thus at low pressure the primary mode of heat transfer across the gap was radiation. The gap was incorporated into the numerical finite volume formulation by the use of a non-uniform node spacing across the thickness of the samples. The location of the nodes was selected so a gap corresponded to an integral number of nodes. The conduction thermal conductivity through the volume elements, defined by the gap nodes, was due to gas conduction only, Equation 13. Within a single layer, it was assumed the combined conduction thermal conductivity of Equation 12 could be used to adequately describe the behavior of the metallic foam.

The solution to the incident radiation equation was applied across the thickness of the sample based on the same non-uniform node spacing used above. However, the density distribution was assumed uniform throughout the sample for the radiation calculations.

The extinction coefficient, albedo of scattering, and the solid conduction efficiency factor are parameters intrinsic to the material under investigation; however, these parameters were not known. Therefore, a subset of measured values was used to predict these parameters based on the Levenberg-Marquardt method for nonlinear parameter estimation.

The subset of measurements chosen for use in the parameter estimation method was selected from the 8 layer sample at $10^{4}$ and $750 \mathrm{~mm} \mathrm{Hg}$. At high vacuum, $10^{-4} \mathrm{~mm} \mathrm{Hg}$, the gas was within the free-molecular regime and conduction through the gas within the void of the foam had a minimal effect. In this regime the contribution of the combined conduction thermal conductivity was expected to have an insignificant influence on the effective thermal conductivity. For this reason, measurements at high vacuum were used to estimate the radiation dependent parameters, $e$ and $\omega$. At high pressure, $750 \mathrm{~mm} \mathrm{Hg}$, the gas was within the continuum regime defined by the temperature dependent thermal conductivity of the gas at atmospheric pressure. In this regime the contribution of the combined conduction thermal conductivity was expected to have a significant contribution to the effective thermal conductivity. The measurements at high pressure were used to estimate the weighting factors for modeling the combined conduction thermal conductivity. 


\section{Numerical Results}

The numerical finite volume formulation produced the same values of the effective thermal conductivities for the 14,11 , and 8 layer samples. Since the numerical results are independent of the number of layers (thus the thickness of the sample) only the 8 layer sample results are presented.

Using the combined conduction thermal conductivity given by Equation 12 in the numerical model produce the curves shown in Figures 8 and 9. The total heat flux through the sample is shown in Figure 8 for the constant temperature difference across the sample of $542 \mathrm{~K}$ and nitrogen gas pressure of $750 \mathrm{~mm} \mathrm{Hg}$. The total heat flux was constant as expected for a steadystate condition. The curves were generated for the 8 layer sample using 5 nodes across each layer. Also shown on the graph are the components of the total heat flux, the heat flux due to combined conduction and the heat flux due to radiation. The discontinuous jumps in heat flux, observable at every fifth node, were due to the presence of gaps between layers. In the figure, the septum plate was located at node position $0.0 \mathrm{~mm}$ and the water-cooled plate was located at $13.69 \mathrm{~mm}$. The dominant mode of heat transfer across the thickness of the sample was combined conduction. Radiation became more significant in the proximity of the septum plate.

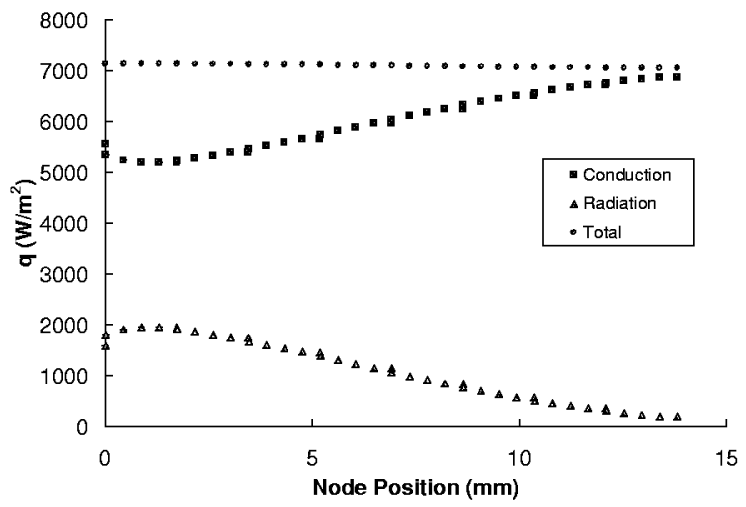

Figure 8. Spatial variation of the total, conductive, and radiative fluxes for the 8 layer sample at $\mathbf{7 5 0}$ mm Hg.

The measured and numerically predicted effective thermal conductivities of the 8 layer sample are shown in Figure 9 for the nitrogen gas pressures of $10^{-4}$ and $750 \mathrm{~mm} \mathrm{Hg}$. The numerical results are presented by the solid curves. The radiation dependent parameters were found to be $e=10.23-1.77 \times 10^{-3} T$ and $\omega=0.8$ where $T$ is the absolute temperature. For $A=1$, analogous to the parallel arrangement, the solid conduction efficiency factor was determined to be $F=0.061$. The identical curve was also produced for $A=0.802$ and $F$ $=0.080$, the parallel-series arrangement. As seen from the figure, the numerical results had excellent correlation to experimental results at low pressure but failed to capture the behavior at high pressure.

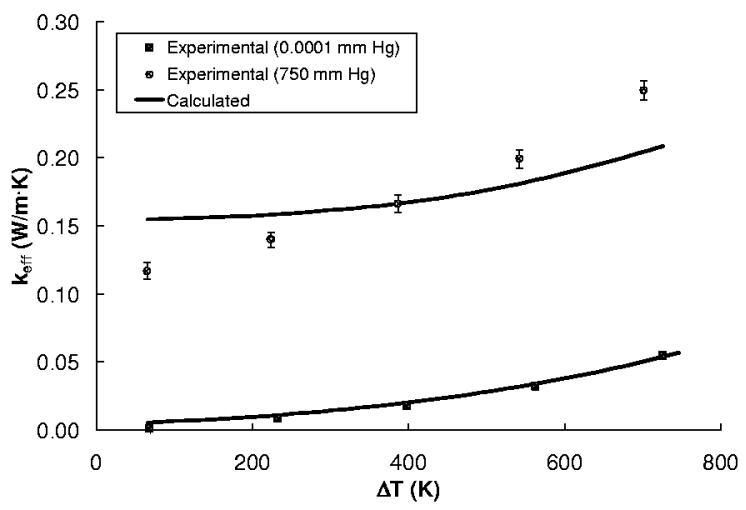

Figure 9. Comparison of measured and predicted effective thermal conductivities using Equation 12 for the combined conduction thermal conductivity.

The difference in effective thermal conductivity between the high pressure and low pressure curves produced by the numerical model was approximately constant while the experimental data had an increase in the difference as the temperature difference across the sample increased. The combined conduction thermal conductivity model given by Equation 12 assumed a superposition of the solid conduction and the gas conduction modes. There must be a coupling or interaction between the solid and gas conduction that is not accounted for in Equation 12. To correct for the discrepancy a coupling term was introduced into the numerical model. The coupling thermal conductivity used, $k_{\text {coupling }}$, is given by

$$
k_{\text {coupling }}=a \cdot\left(k_{\text {gas }} \cdot F \cdot k_{\text {bulk }}\right)^{2}
$$

where $a$ is a coupling weighting factor. Since the thermal conductivity of gas is extremely sensitive to both pressure and temperature, the coupling thermal conductivity is highly dependent on pressure and temperature. The resulting combined conduction thermal conductivity, assuming a parallel arrangement $(A=1)$ in Equation 12, is

$$
k=\varepsilon \cdot k_{\text {gas }}+(1-\varepsilon) \cdot F \cdot k_{\text {bulk }}+a \cdot\left(k_{\text {gas }} \cdot F \cdot k_{\text {butk }}\right)^{2}
$$

When Equation 30 was used for the combined conduction thermal conductivity in the numerical model, the curves shown in Figure 10 were produced. For $\mathrm{A}=1$, the parameter estimation procedure generated the parameters of $F=6.85 \times 10^{-3}$ and $a=$ 389.0. The radiation dependent parameters were determined to be $e=9.85-2.63 \times 10^{-3} T$ and $\omega=0.993$. 
The radiation dependent parameters were slightly affected by the addition of the coupling term since a change in the solid conduction efficiency factor was required. As seen from the figure, the addition of the coupling term placed the numerical results within experimental uncertainties for both the $10^{-4}$ and 750 $\mathrm{mm} \mathrm{Hg}$ regions.

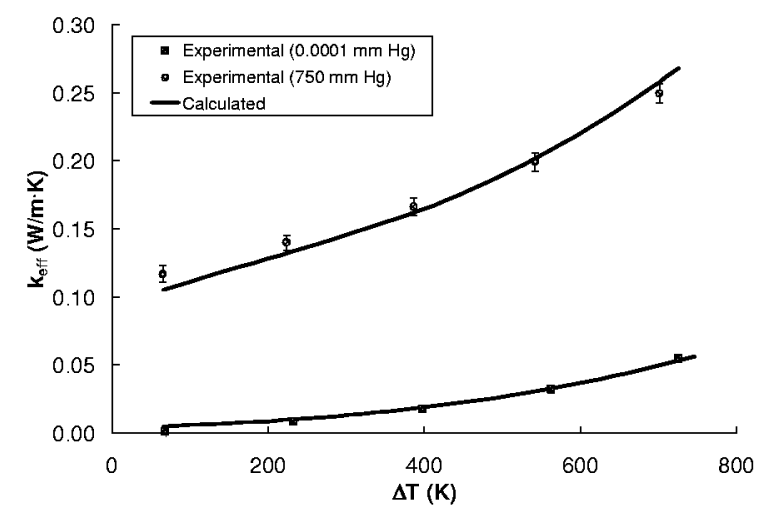

Figure 10. Comparison of measured and predicted effective thermal conductivities using Equation 30 for the combined conduction thermal conductivity.

The gas conduction model given by Equations 13 through 17 was developed for porous media with uniform density fibers or struts. As seen in Figure 2, however, the strut of the metallic nickel foam is hollow. Therefore, the thickness of the strut wall was used in the place of the diameter of the strut in Equation 17. The thickness of the strut wall was approximately 0.014 $\mathrm{mm}$. The calculated values of the effective thermal conductivity for the 8 layer sample with a temperature difference of $500 \mathrm{~K}$ across the thickness of the foam over the pressure range of $10^{4}$ to $750 \mathrm{~mm} \mathrm{Hg}$ are shown in Figure 11. Although the predicted values do not correspond within experimental error limits within the region defined by the pressure of 0.001 to

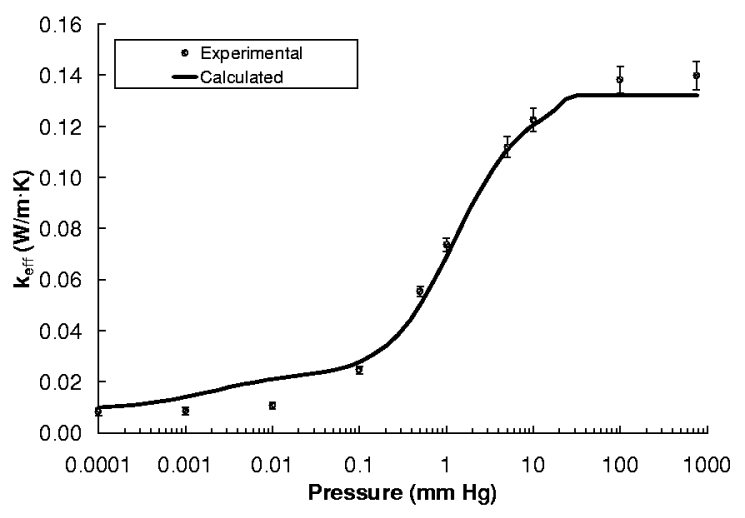

Figure 11. Variation of effective thermal conductivity with pressure.
$0.1 \mathrm{~mm} \mathrm{Hg}$, the predicted values do show the general trend throughout the rarefied, transition, and continuum regions. Neglecting the region between 0.001 and 0.1 $\mathrm{mm} \mathrm{Hg}$, an average 9 percent difference was obtained between the calculated and measured values. The largest percent difference occurred at low pressure, with almost 22 percent difference, and the smallest percent difference occurred at the intermediate pressures, with less than 1 percent difference.

\section{Conclusion}

The effective thermal conductivity of metallic nickel foam was measured for a temperature difference range from 100 to $1000 \mathrm{~K}$ and a pressure range of $10^{-4}$ to 750 $\mathrm{mm} \mathrm{Hg}$. A numerical model was developed to predict the behavior of the effective thermal conductivity at various temperatures and pressures. Using a small subset of experimental data, parameters related to intrinsic properties of the foam were determined. The calculated values of the effective thermal conductivity of the metallic foam produced by the numerical finite volume formulation using the predicted intrinsic parameters were compared to the measured values not used in the parameter estimation method to validate the numerical models. Correlation of the numerical results to the experimental values required the introduction of a conduction coupling term to the gas/solid conduction model. Calculated values corresponded to within an average of 9 percent to the experimental values. The model was consistent with experimental results throughout the environmental conditions under examination.

\section{Acknowledgements}

Research done by the author is a portion of what will be included in a Master of Science Thesis with The George Washington University. The authors would like to thank Mr. Jeffery R. Knutson from NASA Langley Research Center for providing temperature and pressure control and his assistance in conducting the tests, and Mr. James M. Baughman from AS\&M at NASA Langley Research Center for producing the photomicrograph images.

\section{References}

1. Daryabeigi, K., "Design of High Temperature Multilayer Insulation for Reusable Launch Vehicles", Ph.D. dissertation, University of Virginia, May 2000. 
2. Fuganti, A., Lorenzi, L., Hanssen, A.G., and Langseth M., "Aluminum Foams for Automotive Applications," Metal Foams and Porous Metal Structures, ed. by Banhart, J., Ashby, M.F., and Fleck, N.A., Verlag MIT Publishing, 1999.

3. Ettel, V.A., "New Inco Powders and Foams for Nickel Batteries," NiCad 98, Prague, Sept. 21-22, 1998.

4. Kaviany, M., Principles of Heat Transfer in Porous Media, $2^{\text {nd }}$ ed., Springer-Verlag New York, Inc, 1995.

5. Lee, S.-C., and Cunnington, G.R., "Conduction and Radiation Heat Transfer in High Porosity Fiber Thermal Insulation," Journal of Thermophysics and Heat Transfer, Vol. 14, No. 2, April-June 2000, pp.121136.

6. Glicksman, L. R., "Ch. 5, Heat Transfer in Foams," Low Density Cellular Plastics, ed. by Hilyard, N.C., and Cunningham, A., Chapman and Hall, 1994.

7. Gibson, L.J., and Ashby, M.F., Cellular Solids, Structures and Properties - Second Edition, Cambridge University Press Ltd., 1997.

8. Baillis, D., Raynaud, M., and Sacadura, J.F., "Determination of Spectral Radiative Properties of Open Cell Foam: Model Validation", Journal of Thermophysics and Heat Transfer, 14, No. 2, AprilJune 2000.

9. Calmidi, V.V. and Mahajan, R.L., "The Effective Thermal Conductivity of High Porosity Fibrous Metal Foams", ASME Journal of Heat Transfer, 122, May 1999, pp. 466-471.

10. Bhattacharya, A., Calmidi, V.V., Mahajan, R.L., "An Analytical-Experimental Study for the determination of the effective thermal conductivity of high porosity fibrous foams", Application of Porous Media Methods for Engineered Materials, AMD-Vol. 233, ASME 1999, pp. 13-20.

11. Daryabeigi, K., "Analysis and Testing of High Temperature Fibrous Insulation for Reusable Launch Vehicles", 37th AIAA Aerospace Sciences Meeting and Exhibit, AIAA 99-1044, January 11-14, 1999.

12. Tannehill, J.C., Anderson, D.A., and Pletcher, R.H., Computational Fluid Mechanics and Heat Transfer, Second Edition, Tayler \& Francis, 1997, pp. 72.

13. Bauer, T.H., "A General Analytical Approach toward the Thermal Conductivity of Porous Media," Int. J. Heat Mass Transfer, Vol. 36, No. 17, 1993, pp. 4181-4191.

14. Kreith, F. and Black, W.Z., Basic Heat Transfer, Harper \& Row, Publishers, 1980, pp. 509.
15. Pawel, R.E., McElroy, D.L., Weaver, F.J., and Graves, R.S., "High Temperature Thermal Conductivity of a Fibrous Alumina Ceramic," Thermal Conductivity, 19, ed. by David Yarbrough, Plenum Press, New York, 1985, pp. 301-313.

16. M. Necati Özisik, Helcio R. B. Orlande, Inverse Heat Transfer, Taylor \& Francis, 2000, pp. 37.

17. Coleman, H.W., Steele, W.G., Jr., Experimentation and Uncertainty Analysis for Engineers, John Wiley \& Sons, Inc, 1989. 